\title{
Detection and Antibiotic Sensitivity Pattern of Gardnerella vaginalis Isolated from Bacterial Vaginosis Patients Attending Chittagong Medical College Hospital
}

\author{
Nura Nasrin Rowshan Ara ${ }^{1 *}$ \\ Md Anwar Husain² \\ Nasima Akter ${ }^{2}$ \\ Shakeel Ahmed ${ }^{2}$ \\ Md Mostafizur Rahman ${ }^{3}$ \\ Mafruha Nigar ${ }^{4}$ \\ Shamima Akhter ${ }^{2}$
}

\author{
'Department of Microbiology \\ University of Science \& Technology Chittagong (USTC) \\ Chittagong, Bangladesh \\ ${ }^{2}$ Department of Microbiology \\ Chittagong Medical College \\ Chittagong, Bangladesh. \\ ${ }^{3}$ Department of Otolaryngology \\ Cox's Bazar Medical College \\ Cox's Bazar, Bangladesh. \\ ${ }^{4}$ Onestop Crisis Centre \\ Chittagong Medical College Hospital \\ Chittagong, Bangladesh.
}

\begin{abstract}
Background: Now a day's bacterial vaginosis is an extremely common health problem for women in the world which causes many complications both in the pregnancy and non-pregnancy states. G. vaginalis is most important cause of bacterial vaginosis. Methods: A prospective observational study was conducted to detect $G$. vaginalis in bacterial vaginosis and their sensitivity patterns on patients attending at the outpatient Department of Gynaecology and Obstetrics of Chittagong Medical College Hospital, Chittagong. A total of 170 sexually active female in the age group of 15-45 years, with abnormal vaginal discharge were selected for the study. A detailed history and a thorough clinical examination of all the cases were done. Results:In this study $38(22.35 \%)$ Gardnerella vaginalis were isolated by culture and bacterial vaginosis was detected by different methods $47(27.65 \%)$. Antimicrobial resistance is one of the major public health threats. So antimicrobial sensitivity pattern of the organisms should be done at regular intervals. Conclusion: In this study Gardnerella vaginalis showed high (52.63\%) resistant to most commonly used metronidazole and $100 \%$ sensitive to clindamycin.
\end{abstract}

Key words: Bacterial vaginosis; Gardnerella vaginalis; Amsel criteria; Nugent criteria; Human Blood Bilayer Tween 80 (HBT) agar media; Antibiotic sensitivity.

\section{INTRODUCTION}

Bacterial Vaginosis (BV) is a clinical syndrome characterized by shift of protective resident microorganisms as Lactobacillus spp. by opportunistic pathogenic bacteria such as Gardnerella vaginalis and other anaerobic bacteria. In most cases of BV, the predominant bacterial species found is Gardnerella vaginalis. Historically, $G$. vaginalis was thought to be the sole causative agent of this condition. But its role in the aetiology of BV was downgraded over the years. The biofilm-forming potential and cytotoxic activity of $G$. vaginalis have renewed interest in the virulence of this organism $^{1}$. So bacterial vaginosis is mostly caused by the synergistic interaction of $G$. vaginalis with obligate anerobes.

Bacterial vaginosis is associated with many gynaecologic complications, such as cervicitis, salpingitis, endometritis, post-operative infections and pelvic inflmmatory disease, and many obstetric complications, such as premature rupture of the membranes, preterm deliveries, chorioamniotitis and postpartum endometritis. Bacterial vaginosis is also associated with an increased risk of HIV-1 transmission in non-pregnant women and more susceptible to Herpes simplex virus, Chlamydia trachomatis, Neisseria gonorrhoe, and Human Papilloma Virus (HPV) and post surgical infection ${ }^{2}$.

\footnotetext{
Assistant Professor

Department of Microbiology

University of Science \& Technology Chittagong (USTC)

Chittagong, Bangladesh

Mobile: +8801819850606

E-mail: nasrinsumi@yahoo.com
}

Dr. Nura Nasrin Rowshan Ara 
Antibacterial resistance has become a major clinical concern worldwide including Bangladesh ${ }^{3}$. Extensive and indiscriminate use of antibiotics has created a major problemdrug resistance. The widespread and inappropriate use of antibiotics has resulted in the development of a progressively antibiotic-resistant microbial ecosystem in Bangladesh ${ }^{4}$.

This study was designed to isolate the causative agent $G$. vaginalis from bacterial vaginosis patients with their antibiotic sensitivity pattern which would guide clinicians and microbiologists for proper handling of this pathogen \& prevent unnecessary use of antibiotics.

\section{MATERIALS AND METHODS}

This was a prospective observational comparative study carried out in the Department of Microbiology, Chittagong Medical College, Chittagong, during the period of July' 2011 to June 2012. Approval from ethical review committee of Chittagong Medical College was duly taken. A total of 170 women, 50 pregnant and 120 non- pregnant, in the age group of 15-45 years patients attending the Gynae out-patient department of Chittagong Medical College Hospital was enrolled for this study. The results of the experiments were recorded systematically and statistical analysis was done by Statistical Package for Social Sciences (SPSS).

\section{Inclusion criteria:}

Women of reproductive age within 15-45 years, both pregnant and non pregnant, with abnormal vaginal discharge, with or without mild vulver itching or burning are considered as patients.

\section{Exclusion criteria:}

1. Below 15 yr \& over $45 y$ rs.

2. Known case of malignancy or AIDS patient.

3. History of taking antimicrobial agents or vaginal medication for vaginitis within the last one month.

4. Menstruating women.

5. Patient having history of vaginal douche on the day of examination.

\section{Procedure:}

Samples were collected with all aseptic precaution after taking informed consent from patient or her legal attendant. Three vaginal swab samples were collected from each patient by standard technique. First swab sample was collected from right vaginal wall and used for making Gram's stain, amine test and wet mount preparation. Second swab sample was collected from left lateral vaginal wall for culture of Gardnerella vaginalis. Third swab sample collected from vaginal fornix and used for new rapid BV assay test.

\section{Detection of bacterial vaginosis by different clinical and} microbiological methods :-
1. Amsel criteria
2.Nugent criteria
3. Bacterial vaginosis assay test
4. Isolation of Gardenella vaginalis by culture

Culture for isolation of Gardnerella vaginals:

The second swab inoculated into a selective and differential Human Blood Bilayer Tween 80 (HBT) agar media, Human blood agar media, Human blood Colombia agar media for isolation and subculture of $G$. vaginalis.

\section{Procedure of culture:}

Collected vaginal swab was inoculated and the plate was placed immediately in the candle extinction jar containing water soaked cotton. All plates are incubated in $5 \% \mathrm{Co}_{2}$ with increased humidity at $37^{0} \mathrm{C}$ for $48-72 \mathrm{hrs}$ for primary isolation of $G$. vaginalis and read at 48 hours and rechecked at 72 hours before discarded. The plates were examined by oblique lighting after $24 \mathrm{hrs}, 48 \mathrm{hrs}$, and $72 \mathrm{hrs}$.

Colonies on HBT agar media were identified as round opaque, smooth colonies that were pinpoint in size after $24 \mathrm{hrs}$ of incubation and $0.5 \mathrm{~mm}$ in diameter at $48 \mathrm{hrs}$, produce $\beta$ hemolysis after 48 or $72 \mathrm{hrs}$ of incubation.

The $\beta$-hemolytic colonies from HBT agar were examined by Gram's staning to see Gram negative coccobacilli. Subcultures were done on Human blood Columbia agar media and Sheep blood agar media by using $\beta$-haemolytic colony for pure isolation and to see the haemolytic character. Colonies were also used for catalase test, oxidase test and fermentation of different sugar.

The identification of Gardnerella vaginalis, based on:-

1. Colonial morphology: Colonies on HBT agar were identified as small white colonies with $\beta$-hemolysis after 48 to 72 hours of incubation.

2. Clear $\beta$-hemolysis with diffuse edges on HBT media, but no hemolysis on sheep blood agar. The zone of hemolysis was 1 to $2 \mathrm{~mm}$ wide around the isolated colonies on HBT agar after 48 hours of incubation.

3. Gram stained smear from a colony: Gram variable or Gram negative coccobacilli or small rods.

4. Catalase and oxidase test negative.

5. Fermentation of different sugur: - Maltose, mannitol, lactose, sucrose.

6. Susceptibility to different antimicrobial agents.

Antimicrobial susceptibility:-

All the isolates of $G$. vaginalis obtained by culture were tested for antimicrobial susceptibility by the single disc diffusion method against different antimicrobial agents. The organisms were tested against Metronidazole (MTZ) Clindamycine (CD) Ampicilin (AMP) Ceftriaxone (CRO) Erythromycin (E) Ciprofloxacin (CIP) Vancomycin (VA) Cotrimoxazole (SXT) Chloramphenicol (C) Tetracycline (TE).

\section{Reference strain for quality control:-}

The discs from each batch were standardized by testing against reference strains of E. coli ATCC 25922, Staph. aureus 25923 and zones of inhibition were tested with standard value. 


\section{RESULTS}

A total of 170 women, 50 pregnant and 120 non- pregnant, clinically suspected cases of Bacterial Vaginosis (BV) aged between 15-45 years with abnormal vaginal discharge, with or without mild vulver itching or burning were included in this study.

Figure 1 : Out of 170 cases, on the basis of Amsel criteria (Clinical criteria) 43(25.30) cases were Bacterial Vaginosis (BV) positive and $127(74.70 \%) \mathrm{BV}$ negative. On the basis of Nugent criteria 45(26.47\%) were BV positive and $125(73.53 \%)$ BV negative. The results of BV assay test shows 46 (27.06\%) cases were BV assay test positive and rest 124(72.94\%) were negative.

Figure 2 : shows that culture of vaginal fluid yielded growth of G. vaginalis in $38(22.35 \%)$ cases and $132(77.65 \%)$ cases were culture negative.

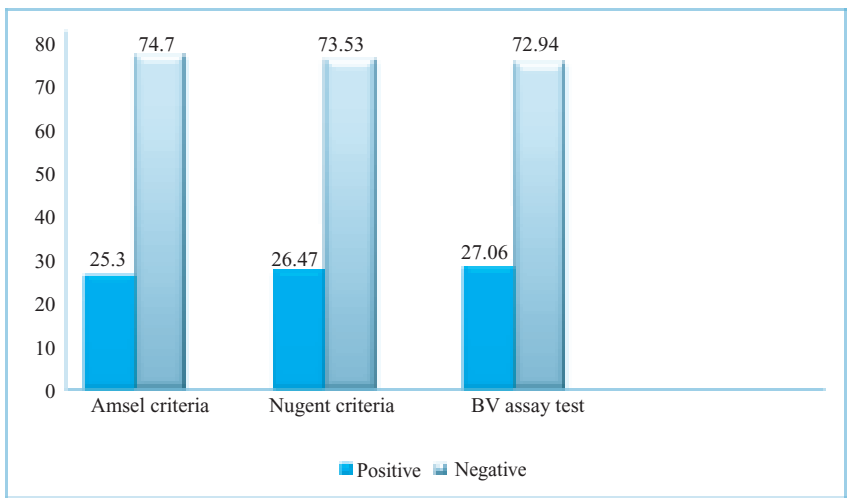

Figure 1: Distribution of bacterial vaginosis by different methods.

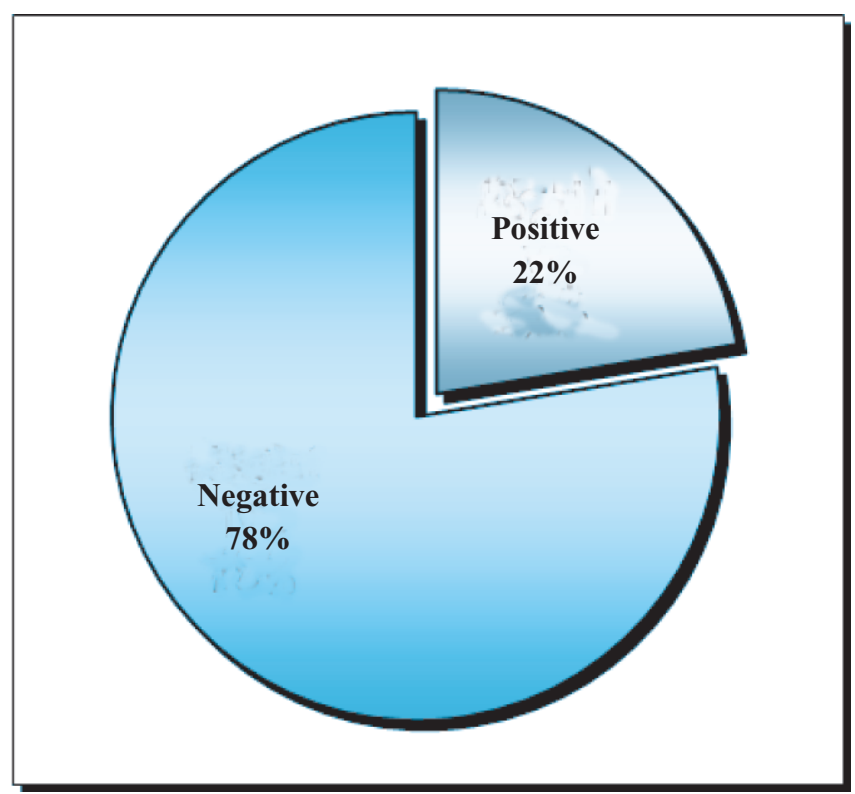

Figure 2 : Distribution of study population on the basis of culture of $G$. vaginalis.

(Pie chart -1: Distribution of culture of GV result)
Table I : The diagnosis of Bacterial Vaginosis (BV) by Amsel criteria, Nugent criteria, BV assay test and culture of $G$. vaginalis are shown in Table I. Out of all 47 BV cases, 38 cases positive by culture were also positive by other three methods. Five more BV cases were positive by these three methods. Additional 02 cases were positive by only BV assay test. One case was positive by both Nugent criteria and BV assay test. Another one case was positive by only Nugent criteria.

Table I : Detection of bacterial vaginosis cases by combined methods $(\mathrm{n}=170)$.

\section{Different Methods \\ BV Positive Percentage (\%)}

Amsel \& Nugent Criteria,

Rapid BV Assay and culture

All four tests positive

Amsel, Nugent Criteria and

Rapid BV Assay

Three tests positive

-culture negative

Nugent Criteria, Rapid BV

Assay test positive \&

Amsel criteria

culture negative

01

Only rapid BV Assay test positive \& other three test negative

Only Nugent Criteria positive \& other three test negative

01

Total (170)

47

27.65

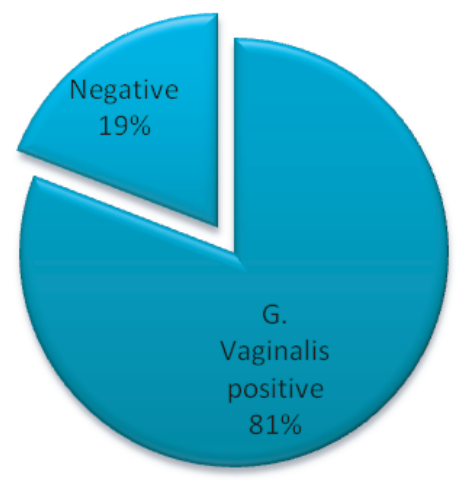

Figure 3 : Distribution of isolated $G$. vaginalis among the total bacterial vaginosis $(\mathrm{n}=47)$

(Pie chart: G. vaginalis in Bacterial vaginosis) 
Table 2 Sensitivity pattern of 38 isolates shows that G. vaginalis were $81.58 \%$ resistant to tetracycline, $78.95 \%$ to cotrimoxazole, $68.42 \%$ to ciprofloxacin, $52.63 \%$ to metronidazole and $26.32 \%$ to erythromycin, while $100 \%$ sensitive to clindamycine followed by vancomycin $(94.74 \%)$ ceftriaxone $(86.84 \%)$ ampicillin $(78.95 \%)$ chloramphenicol $(73.68 \%)$.

Table 2 : Antimicrobial susceptibility pattern of Gardnerella vaginalis isolates $(\mathrm{n}=38)$.

\begin{tabular}{lccr} 
Antimicrobial agent & Sensitive & $\begin{array}{c}\text { Intermediate } \\
\text { sensitive }\end{array}$ & Resistance \\
Clindamycin (CD) & $38(100.00)$ & $00(0.00)$ & $00(0.00)$ \\
Vancomycin (VA) & $36(94.74)$ & $02(5.26)$ & $00(0.00)$ \\
Ceftriaxone (CRO) & $33(86.84)$ & $04(10.53)$ & $01(2.63)$ \\
Ampicillin (AMP) & $30(78.95)$ & $06(15.79)$ & $02(5.26)$ \\
Chloramphenicol (C) & $28(73.68)$ & $06(15.79)$ & $04(10.53)$ \\
Erythromycin (E) & $23(60.52)$ & $05(13.16)$ & $10(26.32)$ \\
Metronidazole (MTZ) & $14(34.21)$ & $04(10.53)$ & $20(52.63)$ \\
Ciprofloxacin (CIP) & $08(21.05)$ & $04(10.53)$ & $26(68.42)$ \\
Cotrimoxazole (SXT) & $04(10.53)$ & $04(10.53)$ & $30(78.95)$ \\
Tetracycline (TE) & $04(10.53)$ & $03(07.89)$ & $31(81.58)$ \\
\hline
\end{tabular}

Figures within parentheses indicate percentages.

\section{DISCUSSION}

Bacterial vaginosis is the most common infection in female worldwide leading to vaginal disorders. It may be a polymicrobial syndrome but recent studies have shown Gardnerella vaginalis (G. vaginalis) also can be a primary pathogen in half of the cases of bacterial vaginosis. This study was primarily designed to detect the G. vaginalis among the BV patients with their sensitivity pattern to decrease the drug resistant.

In the present study, the detection rate of $\mathrm{BV}$ by Amsel criteria, Gram stain Nugent criteria and BV assay test was $43(25.30 \%) 45(26.47 \%)$ and $46(27.06 \%)$ respectively. It correlates with findings of Begum et al, Akhter et al where BV was $24 \%$ and $21.5 \%$ by Amsel criteria, $23 \%$ and $21 \%$ by Gram stain Nugent criteria respectively. On the oher hand, Millar and Posner et $\mathrm{al}^{5-8}$. found bacterial vaginosis by $\mathrm{BV}$ assay test were $39 \%$ and $30 \%$ respectively.

In this study vaginal specimen from study cases were subjected to culture in Human Blood Bilayer Tween (HBT) agar media, a highly selective media, yielded growth of $G$. vaginalis from $22.35 \%$ of total study cases. The isolation was higher than that of Devi et al and Udayalaxmi in India who reported $17.42 \%$ and $16.7 \%$ respectively, but lower than that of Gupta et al in India and and Totten et al in Belgium who reported 54.1\% and 91\% respectively ${ }^{9-12}$. Begum et al, Akhter et al from BSSMU in Bangladesh reported similar findings $25.5 \%$ and $21 \%$ respectively ${ }^{5-6}$.
This slightly higher rate reported by Gupta et al and Totten et al might be due to the use of three or more media that were either non selective or enriched for primary isolation of $G$. vaginalis and variable methods for their identification ${ }^{11-12}$.

In this study total Bacterial Vaginosis (BV) was 47(27.65\%) by Amsel criteria, Nugent criteria, BV assay test and culture of $G$. vaginalis. Out of all $47 \mathrm{BV}$ cases, 38 cases positive by culture were also positive by other three methods. Five more BV cases were positive by these three methods. Additional 02 cases were positive by only BV assay test. One case was positive by both Nugent criteria and BV assay test. Another one case was positive by only Nugent criteria. This findings correlates with the Bilkis et al (22.65\%) Akhter et al (23\%) in Bangladesh and Puri et al (31\%) in India. But Bhalla et al And Mohadani et al found high prevalence $50 \%$ and $51 \%$ respectively ${ }^{13,6,14,15,16}$. Factors responsible for higher prevalence of bacterial vaginosis among the study population were lower socio-economic status, improper sanitation, poor hygiene, malnutrition and might be attributed to non-inclusion of clue cells in their study ${ }^{6}$. Slightly lower incidence in our study may be due to mandatory inclusion of clue cells on saline wet mount and gram stain criteria as a marker of BV for every case, which makes the results more specific.

Gardnerella vaginalis is present in up to $95 \%$ of cases of $\mathrm{BV}^{17}$. But in this study we isolated G. vaginalis $81 \%$ from $\mathrm{BV}$ patient. Gardner \& Dukes isolate G. vaginalis $92 \%$ of woman with BV and Amsel isolated G. vaginalis 96\% from BV patient ${ }^{18,19}$. In our study we used highly selective media, which might be influence the higher growth.

The antibiotic sensitivity pattern of $G$. vaginalis showed extreme variation in different studies. This variation could be attributed to variation in disc potency, media used for susceptibility testing and incubation environment (Aerobic or anaerobic). But there is no difference in opinion regarding use of metronidazole and clindamycin. Both this drugs were found most sensitive in various previous studies ${ }^{13}$. But in our study metronidazole was $52.63 \%$ resistant. Similarly Nagaraja et al and Goldstein et al reported higher resistant to metronidazole ${ }^{20,21}$. Nagaraja reported $68 \%$ resistant to metronidazole. Antibiotic resistance is being increasing day by day around the world.

The sensitivity results show that clindamycin (100\%) vancomycin (94.74\%) and ceftriaxon (86.84\%) were highly sensitive but vancomycin and ceftriaxon were not used for treatment of BV. The next effective drugs were ampicillin $(78.95 \%)$ chloramphenicol $(73.68 \%)$ and erythromycin $(60.52 \%)$. In spite of the higher sensitivity of chloramphenicol and erythromycin, these drugs are not commonly used, because of the toxicity in case of chloramphenicol and inefficiency of erythromycin in acid $\mathrm{pH}$ in vagina ${ }^{22}$. Ampicillin was $78.95 \%$ sensitive against $G$. vaginalis in this study, but was not so effective in vivo. The use of ampicillin for the treatment of 
bacterial vaginosis has often been associated with failure to eradicate G. vaginalis or clinical cure. This is probably due to inactivation of ampicillin by the $\beta$-lactamases produced by vaginal anaerobes. However, this agent may have a role in treating Gardnerella-associated infections at extravaginal sites $^{22}$. Ampicillin also inhibits the growth of Lactobacilli, so prevents recolonization by this organism after therapy ${ }^{23}$.

In our study other effective drug was ciprofloxacin, which was $21.05 \%$ sensitive. As ciprofloxacin is commonly used in UTI, it is expected that women associated with BV could have beneficial effect out of it. Other studies also do not encourage its use in BV.

The maximum resistance found in case of cotrimoxazole (78.95\%) and tetracycline $(81.58 \%)$ in this study, do not recommended its used in $\mathrm{BV}$, though in the past they were used with controversial results ${ }^{11}$.

Finally, several studies have evaluated the clinical and microbiological efficacy of metronidazole and clindamycin to treat $\mathrm{BV}$ and to prevent the recurrence of $\mathrm{BV}^{21,22}$. In our study we found $100 \%$ sensitive to clindamycine but $68 \%$ resistant to metronidazole. So, to know the sensitivity pattern of the organisms at regular interval is important, particularly in developing countries where there is excessive use of antibiotics and lack of adequate antimicrobial resistance surveillance ${ }^{3}$.

\section{CONCLUSION}

Though bacterial vaginosis is a global problem, we should detect the principle pathogen and treat them properly. Extensive and indiscriminate use of antibiotics has created a major problem- drug resistance. Several antimicrobial agents have been used to treat symptomatic BV. Until recently, the mainstay therapy consisted of either metronidazole or clindamycin. But in our study Gardnerella vaginalis showed high resistance to commonly used antibiotics metronidazole. So indiscriminate use of antibiotics should be avoided.

\section{DISCLOSURE}

All the authors declared no competing interest. 


\section{REFERENCES}

1. Patterson, JL, Stull-lane, A, Girerd, PH \& Jefferson, KK. Analysis of adherence, biofilm formation and cytotoxicity suggests a greater virulence potential of Gardnerella vaginalis relative to other bacterial-vaginosis-associated anaerobes. Microbiology. 2010; 156(2):392-399.

2. Watts, DH, Fazzari, M, Minkoff, H, Hillier, SL, Sha, B, Glesby, M, Levine, AM \& Burk, R. Effects of bacterial vaginosis and other genital infections on the natural history of human papillomavirus infection in HIV-1-infected and high-riswomen .J Infect Dis. 2005; 191: $1129-1139$

3. Karim S, Ahmed S, Parvez M, Mottalib M, Islam AHMS, Rahman M \& Haq JA. Emerging multi-drug resistant organisms in a tertiary care hospital of Dhaka city. Bangladesh J of Medical Science. 2002; 8(1): 53-7.

4. Faiz MA \& Basher A. Antimicrobial resistance: Bangladesh experience. Regional Health Forum. 2011; 15(1): 1-8.

5. Begum, N, Muazzam, N, Shamsuzzaman, SM, Islam, MDU, Chowdhury, AK \& Rashid A. Diagnosis of bacterial vaginosis by acridine orange staining and its comparison to conventional methods and association of Gardnerella vaginalis with bacterial vaginosis. Bangladesh $J$ Med Microbiol. 2010; 04(01): 37-42

6. Akhter S, Satter H,Tarafder S, Miah RA, Sharmin S, Ahmed S. Bangladesh J Med Microbiol. 2010; 04 (01): $24-27$.

7. Miller, L. Can Fem Exam card use facilitate bacterial vaginosis diagnosis on data of abortion to prevent postabortion endometritis. The American College of Obstetricians \& Gynecologists. 2001; 97(4) : S58-S59.

8. Posner, SF, Kerimova, J, Aliyeva, F \& Duerr, A. Strategies for diagnosis of bacterial vaginosis in a resource - poor setting. International J of STD \& AIDS. 2005;16: 52-55.

9. Devi, PS, Rao, Ashriyan, M, Rajaram, K \& Jagadish, C. Diagnosis of Bacterial vaginosis in a rural setup. Indian J of Medical Microbiology. $2004 ; 22,(1): 47-50$

10. Udayalaxmi, G, Subbannayya, K \& Shalini, S. Comparison of the methods of bacterial diagnosis of bacterial vaginosis. $J$ of $C l i n i c a l$ and Diagnostic Research. 2011; 5: 498-501.

11. Gupta, BK, Kumar, R, Sofat, R, Khurana, S \& Deepinder. The role of Gardnerella vaginalis in non-specific vaginitis in intrauterine contraceptive device user. Indian J of pathology and microbiology. 1998; 41(1):67-70.

12. Totten, PA, Amsel, R, Hale, J, Piot, P \& Holmes, KK. Selective defferential human blood bilayer mediafor isolation of Gardnerella (Haemophilus) vaginalis. J Clin Microbiol. 1982; 15: 141-47.

13. Bilkis, B. Rapid laboratory diagnosis of BV by New prolin Aminopeptidase assay and comparison with conventional methods. M.Phil.Thesis Department of microbiology Bangabandhu Sheikh Mujib Medical University, Dhaka, Bangladesh. 2003.

14. Puri, KJ, Madan, A \& Bajaj, K. Incidence of various causes of vaginal discharge among sexually active females in age group $20-40$ years. Indian J Dermatol Venereol Leprol. 2003; 69:122-125.

15. Bhalla P, Kaushika A. Epidemilogical and microbiological correlates of bacterial vaginosis. Indian J Dermatol Venereol Leprol. 1994; 60:8-14.

16. Mohadani JW, Dekate RR, Shrikhande AV. Cytodiagnosis of discharge per vaginum. Indian J Pathol Microbiol. 1998; 41:403-411.

17. Marrazzo, JM, Thomas, KK, Fiedler, TL, Ringwood, K \& Fredricks, DN. Relationship of specific vaginal bacteria and bacterial vaginosis treatment failure in women who have sex with women. Ann Intern Med. 2008;149; 20-28.

18. Gardner, HL \& Dukes, CD. Haemophilus vaginalis vaginitis: A newly defined specific infection previously classified non-specific vaginitis Am J Obstet Gynecol. 1955; 69: 962-976.

19. Amsel, R, Totten, PA, Spiegel, CA, Chen, KCS, ES chenbach, D \& Holmes, KK. Nonspecific vaginitis: Diagnostic criteria and microbial and epidemiological association. Am J Med. 1984; 74: 14-22.

20. Nagaraga, P. Antibiotic resistance of Gardnerella Vaginalis in recurrent bacterial vaginosis. Indian J of Medical Microbiology. 2008; 26(2):155-157.

21. Goldstein, EJC, Citron, DM, Merriam, CV, Warren, YA, Tyrrell, KL \& Fernandez, HT. In vitro activities of Garenoxacin (BMS 284756) against 108 clinical isolates of Gardnerella vaginalis. Antimicrob agents chemother. 2002; 46(12): 3995-3996.

22. Kharsany, ABM, Hoosen, AA \& DenEnde, JV. Antimicrobial susceptibilities of Gardnerella vaginalis. Antimicrob Agents Chemother. 1993; 37(12): 2733-2735

23. Spiegel, CA. Bacterial vaginosis. Clinical microbiology reviews. 1991; 4(4): 485-502 